\title{
Adherencia al tratamiento ortodóncico de pacientes adolescentes atendidos en una institución docente asistencial de Medellín. Estudio transversal
}

Adherence to the orthodontic treatment of adolescent patients seen in a teaching institution in Medellin. Transversal study

Adesão ao tratamento ortodôntico de pacientes adolescentes atendidos em uma instituição de ensino de Medellín. Estudo transversal

\section{Juliana Cano Restrepo ${ }^{1}$ Cecilia María Martínez Delgado²}

Recibido: 14 de junio del 2018 Aprobado: 28 de agosto del 2018 Publicado: 20 de marzo de 2019

Cómo citar este artículo: Cano- Restrepo J., Martínez- Delgado C. M. Adherencia al tratamiento ortodóncico de pacientes adolescentes atendidos en una institución docente asistencial de Medellín. Estudio transversal. Revista Nac Odontol. 2019; 15(28): 1-19. doi: https://doi.org/10.16925/2357-4607.2019.01.01

Artículo de investigación. https://doi.org/10.16925/2357-4607.2019.01.01

1 Universidad CES, Medellín, Colombia

https://orcid.org/0000-0001-9718-3533

2. Universidad CES, Medellín, Colombia.

https://orcid.org/0000-0001-8409-7337

cmariamar@hotmail.com 


\section{Resumen}

Introducción: La adherencia a un tratamiento es una condición indispensable para el éxito del mismo. Es un fenómeno multidimensional determinado por la acción recíproca de varios factores.

Objetivo: Identificar la adherencia al tratamiento ortodóncico de pacientes adolescentes atendidos en una institución docente asistencial de Medellín (Colombia), en el año 2016.

Materiales y métodos: Estudio transversal con una muestra intencionada de 101 pacientes entre 12 y 18 años, de ambos sexos, residentes en Medellín y área metropolitana, que recibieron tratamiento de ortodoncia. El proyecto fue aprobado por el Comité de Ética, mediante acta No. 77 de febrero de 2015. Se modificaron los cuestionarios propuestos por Haynes y Sackett (1980) y Morisky, Green y Levine (1986), adaptando cada pregunta a la terminología relacionada con el tratamiento ortodóncico. Dichos cuestionarios miden la adherencia de pacientes a los tratamientos que requieren medicamentos. Se realizó un análisis estadístico univariado y bivariado (prueba Chi2 de independencia y ANOVA).

Resultados: El 86,1 \% de los encuestados recibieron instrucciones sobre cuidado bucal, pero solo el $57,4 \%$ las recuerdan; el $89,1 \%$ asiste a las citas. El $45.5 \%$ de los pacientes mostraron alta y baja adherencia al tratamiento de ortodoncia y el $8.9 \%$ son fueron adherentes.

Conclusión: En igual porcentaje se encontró adherencia alta y baja. Ambos porcentajes no son satisfactorios, por tanto, es necesario redoblar esfuerzos para mejorar un indicador tan importante para el bien de los pacientes y la imagen institucional.

MeSH: Necesidades de tratamiento ortodóncico, Técnicas de movimiento dental, Adherencia al tratamiento, Maloclusión, Comportamiento adolescente.

\section{Summary}

Introduction: The adherence to a treatment is an indispensable condition for its success. It is a multidimensional phenomenon determined by the reciprocal action of several factors.

Objective: To identify the adherence to orthodontic treatment of adolescent patients attended in a teaching institution of Medellín (Colombia), in 2016.

Materials and methods: A cross-sectional study with an intentional sample of 101 patients between 12 and 18 years old, of both sexes, residents of Medellín and the metropolitan area, who received orthodontic treatment. The project was approved by the Ethics Committee, by means of act No. 77 of February 2015. The questionnaires proposed by Haynes and Sackett (1980) and Morisky, Green and Levine (1986) were modified, adapting each question to the terminology related to orthodontic treatment These questionnaires measure the adherence of patients to treatments that require medication. A univariate and bivariate statistical analysis was performed (Chi2 test of independence and ANOVA).

Results: $86.1 \%$ of the respondents received instructions on oral care, but only $57.4 \%$ remember them; $89.1 \%$ attend appointments. $45.5 \%$ of patients showed high and low adherence to orthodontic treatment and $8.9 \%$ were adherent.

Conclusion: High and low adherence were found in the same percentage. Both percentages are not satisfactory, therefore, it is necessary to redouble efforts to improve such an important indicator for the good of the patients and the institutional image.

MeSH: Orthodontic treatment needs, Dental movement techniques, Adherence to treatment, Malocclusion, Adolescent behavior. 


\section{Resumo}

Introdução: A adesão a um tratamento é condição indispensável para o seu sucesso. É um fenômeno multidimensional determinado pela ação recíproca de vários fatores.

Objetivo: Identificar a adesão ao tratamento ortodôntico de pacientes adolescentes atendidos em uma instituição de ensino de Medellín (Colômbia), em 2016.

Materiais e métodos: Estudo transversal com amostra intencional de 101 pacientes entre 12 e 18 anos, de ambos os sexos, residentes de Medellín e região metropolitana, que receberam tratamento ortodôntico. 0 projeto foi aprovado pela Comissão de Ética, pela Lei No. 77 de Fevereiro de 2015. questionários propostos por Haynes e Sackett (1980) e Morisky, verde e Levine (1986) foram modificados, adaptando cada terminologia pergunta relacionada tratamento ortodôntico Esses questionários medem a adesão dos pacientes aos tratamentos que requerem medicação. Foi realizada uma análise estatística univariada e bivariada (teste de independência de Chi2 e ANOVA).

Resultados: $86,1 \%$ dos entrevistados receberam instruções sobre higiene bucal, mas apenas $57,4 \%$ lembram deles; $89,1 \%$ comparecem às consultas. $45,5 \%$ dos pacientes apresentaram alta e baixa adesão ao tratamento ortodôntico e 8,9\% eram aderentes.

Conclusão: Alta e baixa adesão foram encontradas no mesmo percentual. Ambos os percentuais não são satisfatórios, portanto, é necessário redobrar os esforços para melhorar um indicador tão importante para o bem dos pacientes e a imagem institucional.

MeSH: Necessidades de tratamento ortodôntico, Técnicas de movimentação dentária, Aderência ao tratamento, Má oclusão, Comportamento do adolescente.

\section{Introducción}

Uno de los retos más importantes, cuando se instaura un tratamiento médico u odontológico, es lograr y mantener alta adherencia, especialmente en adolescentes. Este grupo de edad es importante en el análisis de los servicios de salud por cuanto es una época de la vida en la que suceden duelos y etapas que definen en un futuro los rasgos de personalidad y de carácter, de comportamientos desarrolladores y afianzadores de la relación consigo mismo y con los demás (1).

Este periodo de la vida es uno de los momentos propicios para recurrir al tratamiento de ortodoncia, tanto para lograr una alineación funcional de los dientes como por estética (2). Dicho tratamiento puede producir incomodidades como dolor, dificultad para comer, restricción de ciertos alimentos, de tal manera que se requiere un apoyo constante de los padres para que los jóvenes asuman con responsabilidad y compromiso su tratamiento $(3,4)$.

El término adherencia a un tratamiento ha pasado por diferentes acepciones. Una de ellas es "compliance" (conformidad), propuesta por Sackett (1976), quien posteriormente con Haynes (1978), definieron el término como "el grado en que el 
comportamiento de una persona, llámese, toma del medicamento, seguimiento de una dieta o modificaciones del estilo de vida, coinciden con el consejo médico o de otro profesional de la salud" (5).

En los años ochenta se empieza a utilizar el término "cooperación" y con él, su relación con el paciente, relación que puede verse afectada por múltiples factores que, sumados al compromiso o autocuidado, la complejidad del tratamiento, el cumplimiento de las citas, la higiene oral, el tiempo y duración del tratamiento, entre otros, afectarán su adecuada evolución $(6,7)$.

El término "cooperación" cambió y se transformó en "adherencia" introducido por Kontz en el año 1991 (8). La Organización Mundial de la Salud (OMS) en 2004 la define como un fenómeno multidimensional, determinado por la acción recíproca de cinco parámetros, a saber: factores relacionados con el tratamiento, factores relacionados con el paciente, factores relacionados con la enfermedad, factores relacionados con el sistema de atención sanitaria y factores socioeconómicos. Todos ellos y probablemente otros que no se han investigado, interactúan para hablar de multidimensionalidad del fenómeno (9).

Adherencia en ortodoncia significa acudir a las citas programadas, mantener una buena higiene oral, el uso de elásticos o aparatos funcionales como le indica el clínico y evitar los alimentos que pueden provocar la caída de los brackets entre otros (10). Una causa adicional, y probablemente la más grave y frecuente en el paciente, es el olvido (asistencia a citas de seguimiento y control, colocar los aditamentos, uso de los elementos de higiene oral) $(10,11)$

Kgatlwane y cols (2005) sustentan que el comportamiento humano y las creencias son también factores críticos a considerar: inadecuados conocimientos y actitudes negativas hacia el tratamiento, sus efectos secundarios, las limitaciones financieras, los factores relacionados con el servicio, la discriminación y diversas razones socioculturales, pueden ser posibles causas de la no adherencia al tratamiento $(12,13)$ y aumentar el costo de la atención y los malos resultados $(14,15)$.

En la práctica del ortodoncista lo que más se observa empíricamente durante un tratamiento de ortodoncia es que el éxito o fracaso de éste se puede asociar a la sumatoria de factores descritos anteriormente (16), con una proporción de deserción del tratamiento hasta del 21.4 \% (4). Esta variabilidad hace que los pacientes presenten inconvenientes en mantener su tratamiento de manera apropiada; vale decir, cumplimiento de todas y cada una de las recomendaciones del clínico (15,17-19).

Existen diferentes formas de valorar la adherencia a los tratamientos (20-22). Autores como Haynes y Sackett (1980) y Morinsky, Green y Levine (1986) propusieron diferentes cuestionarios, los cuales se utilizan con mayor frecuencia en la 
práctica clínica médica debido a su alta especificidad, sencillez y brevedad $(23-25,18)$. En odontología no hay muchos estudios sobre adherencia a los tratamientos. Martínez y cols. (2014) aplicaron dicho cuestionario para evaluar la adherencia a un tratamiento preventivo de enfermedades bucales inducidas por placa bacteriana mediante gomas de mascar sin azúcar, en pacientes jóvenes, con resultados favorables (26).

A pesar del gran interés por el tema, en la actualidad hay varias formas de medir la adherencia, por lo que se pueden hacer de manera individual o combinada con el fin de corregir las insuficiencias que los instrumentos puedan poseer (18): 1) Los métodos objetivos directos se basan en la determinación del fármaco, de sus metabolitos o de sustancias trazadoras en algún fluido biológico, frecuentemente sangre u orina. 2) Los métodos objetivos indirectos valoran el incumplimiento a partir de circunstancias relacionadas y con el grado de enfermedad (27). 3) Los métodos subjetivos son sencillos y económicos, y los más adecuados cuando la población que se valora es amplia. Entre ellos figuran el juicio del médico y las técnicas de entrevista (28).

La literatura describe varios cuestionarios para la evaluación de la adherencia (cumplimiento terapéutico). Cabe destacar los dos más importantes: Test de Morinsky Green y Levine y el de Haynes-Sackett. $(23,24)$. El primero, validado para diversas enfermedades crónicas, fue desarrollado originalmente por estos tres autores en los años 80 para valorar el cumplimiento de la medicación en pacientes con hipertensión arterial (HTA). También se ha usado en la valoración del cumplimiento terapéutico en diferentes enfermedades $(20,21,29)$.

El propósito de este estudio fue identificar la adherencia al tratamiento ortodóncico de pacientes adolescentes atendidos en una institución docente asistencial de Medellín, explorando variables que reporta la literatura como probables trazadoras de la falta de adherencia para tratar de comprender un fenómeno complejo y brindar a la comunidad académica y a los clínicos datos relevantes que permitan garantizar, en la medida de lo posible, seguimiento y motivación a los pacientes y su entorno familiar, en beneficio de ellos mismos y de los servicios de salud.

\section{Materiales y métodos}

Se realizó un estudio descriptivo transversal con una muestra por conveniencia de 101 pacientes, residentes en Medellín y área metropolitana, que reciben tratamiento de ortodoncia en la IPS Clínica CES (Sabaneta), previa aprobación del Comité de Ética y de Investigación, mediante acta No. 77 de febrero de 2015. Los pacientes se reclutaron durante dos meses (diciembre de 2015 y enero de 2016). 
Los criterios de inclusión fueron: pacientes sistémicamente sanos que llevasen seis (6) meses o más de tratamiento, de cualquier sexo, en las edades comprendidas entre 12 y 18 años de edad, que residieran en la ciudad de Medellín (Colombia) o en el área metropolitana. A los entrevistados se les explicó el propósito del estudio y todos leyeron el asentimiento informado (menores de edad), luego de lo cual dieron su aceptación para participar en el estudio. Los padres, acudientes o testigos, firmaron el consentimiento informado (Resolución 008430 de 1993 expedida por el anterior Ministerio de Salud de Colombia). Se protegió la confidencialidad de los datos, mediante el anonimato de la encuesta. Es una investigación sin riesgo.

Los cuestionarios propuestos por Haynes y Sackett (1980) y Morisky, Green y Levine (1986), ambos validados para medir la adherencia de los pacientes a los tratamientos con medicamentos, se modificaron adaptando cada pregunta a la terminología relacionada con el tratamiento ortodóncico. El cuestionario quedó integrado por 26 preguntas (tabla 1), previa aplicación de prueba piloto.

La encuesta fue aplicada por la investigadora principal, con el apoyo de estudiantes de pregrado de odontología de la Universidad CES, pertenecientes al semillero de investigación, los cuales fueron entrenados en las preguntas y en el propósito de ellas.

Tabla 1. Comparación de los test de Morinsky-Green-Levine, de Haynes y Sackett y del presente estudio

\begin{tabular}{|c|c|c|}
\hline $\begin{array}{c}\text { Test de } \\
\text { Morinsky-Green-Levine. } \\
\text { Consta de } 4 \text { preguntas } \\
\text { (todas Sí o No) }\end{array}$ & $\begin{array}{l}\text { Cuestionario de comunicación } \\
\text { de autocumplimiento } \\
\text { de Haynes y Sackett. } \\
\text { Consta de } 3 \text { preguntas }\end{array}$ & $\begin{array}{l}\text { Cuestionario adherencia } \\
\text { tratamiento ortodóncico. } \\
\text { Consta de } 11 \text { preguntas }\end{array}$ \\
\hline $\begin{array}{l}\text { 1. ¿Olvida tomar alguna vez } \\
\text { los medicamentos? }\end{array}$ & $\begin{array}{l}\text { 1. La mayoría de las personas, por } \\
\text { una u otra razón tienen dificultad } \\
\text { en tomar comprimidos. ¿Tiene } \\
\text { usted dificultad en tomar todos } \\
\text { los suyos? }\end{array}$ & $\begin{array}{l}\text { 1. ¿Su odontólogo tratante le dio } \\
\text { recomendaciones adicionales de } \\
\text { higiene oral? (cepillos especiales, } \\
\text { enebradores, enjuagues) } \\
\text { Si:___No:____ }\end{array}$ \\
\hline $\begin{array}{l}\text { 2. ¿Descuida la hora en } \\
\text { la cual debe tomar la } \\
\text { medicación? }\end{array}$ & $\begin{array}{l}\text { 2. Para los que respondan que no } \\
\text { tienen dificultad, se insiste: ¿Los } \\
\text { toma? } \\
\text { Todos los días___ Muchos días___ } \\
\text { Algunos días___ }\end{array}$ & $\begin{array}{l}\text { 2. ¿Se acuerda de las instrucciones? } \\
\text { Si:__ No: _- }\end{array}$ \\
\hline $\begin{array}{l}\text { 3. ¿Cuándo se siente } \\
\text { bien deja de tomar la } \\
\text { medicación? }\end{array}$ & $\begin{array}{l}\text { 3. Muchas personas tienen dificultad } \\
\text { en seguir los tratamientos. ¿Por qué } \\
\text { no me comenta cómo le va a usted? } \\
\text { (Recoger lo que diga el paciente) }\end{array}$ & $\begin{array}{l}\text { 3. ¿Llega a la cita el día indicado por } \\
\text { su ortodoncista? } \\
\text { Si:__ No: __ }\end{array}$ \\
\hline $\begin{array}{l}\text { 4. ¿Si alguna vez le cae } \\
\text { mal deja de tomar la } \\
\text { medicación? }\end{array}$ & & $\begin{array}{l}\text { 5. ¿Cuándo cree que ya está bien } \\
\text { el tratamiento, deja de asistir } \\
\text { a las citas? } \\
\text { Si:__No: __ }\end{array}$ \\
\hline
\end{tabular}




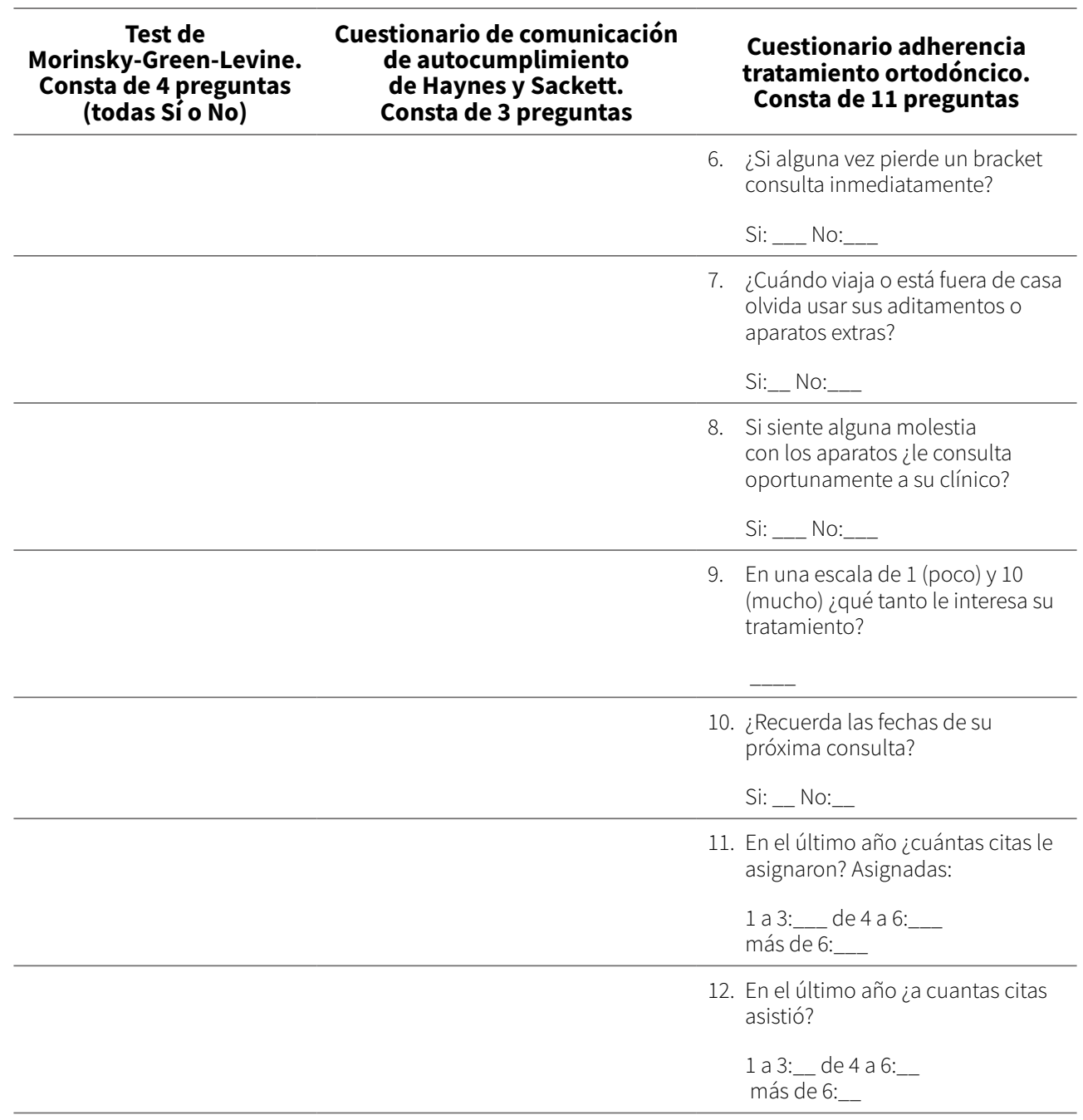

Fuente: Elaboración propia

Las variables estudiadas se agruparon en 3 componentes: 1) Sociodemográficas como edad, sexo, estrato, procedencia, afiliación a la seguridad social y pago del tratamiento. 2) Variables clínicas como autocuidado bucal (higiene y hábitos) y tipos de aparatología. 3) Variables relacionadas directamente con la adherencia (11 preguntas), además de que se buscaron explicaciones para comprender las motivaciones que pudieran acompañar las respuestas positivas o negativas de los pacientes a cada una de las preguntas que ameritaron explicación adicional. 
Adherencia al tratamiento ortodóncico de pacientes adolescentes atendidos en una institución docente asistencial de Medellín. Estudio transversal

\section{Control de sesgos}

Los sesgos de información se controlaron con la elaboración de un instructivo para el diligenciamiento de la encuesta, siempre apoyados por los investigadores. La prueba piloto, y la posterior discusión de las preguntas con los encuestadores, permitieron obtener respuestas veraces en la mayoría de los encuestados. No existió un proceso de selección aleatoria que garantizara el control de los sesgos de selección, sin embargo, el desconocimiento de la identidad de los pacientes, además del tamaño muestral y la manera como se contactaron los participantes permitió este control.

\section{Análisis estadístico}

Los datos se organizaron en el programa Excel® para Windows (2010); se procedió a la limpieza de la información recolectada y posteriormente se tabuló en el programa SPSS® (IBM SPSS Statistics 19.0.1 - diciembre 2010)

Las variables relacionadas con adherencia se recodificaron dándoles un valor de 0 (no adherencia), 0,5 (adherencia baja) y 1 (alta adherencia), según lo propusieron los autores de referencia. El análisis univariado se expresó en promedio y desviación estándar para la edad. Las demás variables fueron cualitativas, utilizando para su descripción las frecuencias absolutas y relativas.

Se realizó análisis bivariado por edad, sexo, estrato socioeconómico, procedencia y tipo de aparatología como variables independientes, en relación con el nivel de adherencia (variable desenlace), asumiendo una significancia estadística $p<0,05$. Para ello se utilizaron la prueba Chi2 de independencia para asociar las variables cualitativas y ANOVA para comparar la edad de los encuestados respecto a su nivel de adherencia.

\section{Resultados}

Se incluyeron en el estudio 101 pacientes que voluntariamente aceptaron diligenciar la encuesta. La tabla 2 resume las principales características sociodemográficas de los sujetos estudiados. La edad de los entrevistados estuvo entre 12 y 18 años, por cuanto el estudio consideró entrevistar a adolescentes en este rango de edad; el mayor aporte lo hicieron las mujeres (54\%), en su mayoría cursaban estudios secundarios y se clasificaron en un estrato socioeconómico medio (3 y 4 de la clasificación del SISBEN).

Aunque en este estudio se preguntó por la procedencia, en Medellín y el área metropolitana no puede hablarse en sentido estricto de áreas rurales por la cercanía con la ciudad, por el acceso a los servicios y por las características culturales. Los resultados informaron que un 92,1 \% (n=93) viven o proceden del área urbana. 
Tabla 2. Características sociodemográficas

\begin{tabular}{lcc}
\hline Variables & n & Promedio ( \pm d.s.) \\
\hline Edad & 101 & $15,2 \pm 1,6$ \\
\hline
\end{tabular}

\begin{tabular}{lccc}
\hline & & $\mathbf{n}$ & Frec. Relativa (\%) \\
\hline Sexo & Masculino & 47 & 46,5 \\
\hline & Femenino & 54 & 53,5 \\
\hline & & & \\
\hline \multirow{2}{*}{ Escolaridad } & Primaria & 2 & 2 \\
\cline { 2 - 4 } & Secundaria & 88 & 87,1 \\
\cline { 2 - 4 } & Universitaria & 10 & 9,9 \\
\cline { 2 - 4 } & SD & 1 & 1 \\
\hline
\end{tabular}

\begin{tabular}{cccc}
\hline & 1 & 2 & 2 \\
\cline { 2 - 4 } Estrato & 2 & 25 & 24,8 \\
\cline { 2 - 4 } & 3 & 47 & 46,5 \\
\hline & 4 & 13 & 12,9 \\
\hline 5 & 9 & 8,9 \\
\hline & No sabe & 4 & 4 \\
\hline
\end{tabular}

\begin{tabular}{lccc}
\hline \multirow{2}{*}{ Procedencia } & Urbana & 93 & 92,1 \\
\cline { 2 - 4 } & Rural & 8 & 7,9 \\
\hline
\end{tabular}

\begin{tabular}{lccc}
\hline & Sí & 99 & 98,0 \\
\cline { 2 - 4 } Afiliación a SS* & No & 1 & 1,0 \\
\cline { 2 - 4 } & No sabe & 1 & 1,0 \\
\hline
\end{tabular}

\begin{tabular}{lccc}
\hline & Padre & 40 & 39,6 \\
\cline { 2 - 4 } Responsable pago** $^{*}$ Madre & 26 & 25,7 \\
\cline { 2 - 4 } & Ambos & 22 & 21,8 \\
\cline { 2 - 4 } & Familiar & 11 & 10,9 \\
\cline { 2 - 4 } & Otro & 2 & 2 \\
\hline
\end{tabular}

* Afiliación al Sistema General de Seguridad Social en Salud

** Responsable del pago del tratamiento del paciente

Fuente: Elaboración propia 
Adherencia al tratamiento ortodóncico de pacientes adolescentes atendidos en una institución docente asistencial de Medellín. Estudio transversal

La tabla 3 describe las variables clínicas contempladas en el estudio; un alto porcentaje $(60,4 \% ; n=61)$ recurrió al tratamiento de ortodoncia por cuanto presentaba maloclusión; un porcentaje menor (26,7 \%) informó solicitar el tratamiento por estética.

Tabla 3. Características clínicas

\begin{tabular}{lllc}
\hline Variables & n & Frec relativa (\%) \\
\hline Por qué tratamiento & Maloclusión & 61 & 60,4 \\
\hline & Estética & 27 & 26,7 \\
\hline & Indicaciones profesional & 6 & 5,9 \\
\hline & Otras respuestas & 6 & 5,9 \\
\hline & SD & 1 & 1,0 \\
\hline Tipo de tratamiento & & & \\
\hline & Roth & 76 & 75,2 \\
\hline & MBT & 4 & 4 \\
\hline & Damon & 6 & 5,9 \\
\hline Uso aparatos extras & Estándar & 15 & 14,9 \\
\hline & Sí & 43 & 42,6 \\
\hline
\end{tabular}

\begin{tabular}{llcc}
\hline Frec. cepillo dental & 1 vez/día & 1 & 1,0 \\
\hline 2 veces/día & 25 & 24,8 \\
\hline 3 veces/día & 60 & 59,4 \\
\hline 4 veces/día & 12 & 11,9 \\
\hline Otros & 3 & 3,0 \\
\hline
\end{tabular}

\begin{tabular}{llll}
\hline Frec. seda dental & No usa & 37 & 36,6 \\
\hline 1 vez/día & 34 & 33,7 \\
\hline 2 veces/día & 17 & 16,8 \\
\hline 3 veces/día & 12 & 11,9 \\
\hline 4 veces/día & 1 & 1,0 \\
\hline
\end{tabular}

\begin{tabular}{llll}
\hline Frec. Enjuagues & No usa & 47 & 46,5 \\
\hline 1 vez/día & 23 & 22,8 \\
\hline 2 veces/día & 18 & 17,8 \\
\hline 3 veces/día & 13 & 12,9 \\
\hline
\end{tabular}

Fuente: elaboración propia 
Los tratamientos ortodóncicos tradicionalmente utilizados en los servicios asistenciales son de tipo Roth y Estándar. Efectivamente los resultados indicaron que el $75,2 \%$ ( $n=76)$ y el 14,9 \% ( $n=15)$ respectivamente, tuvieron estos tipos de tratamiento.

El uso de aditamentos extra a los bracketts es decisión del clínico de acuerdo al tipo de maloclusión que tenga el paciente; no necesariamente es obligatorio utilizarlos, por ello, el mayor porcentaje de los entrevistados reportó no tenerlos prescritos por el especialista ( $n=58 ; 57,4 \%)$.

Las recomendaciones de higiene bucal y su seguimiento estricto son muy importantes para el éxito del tratamiento. En el presente estudio se encontró que más de la mitad de los pacientes ( $n=60 ; 59,4 \%$ ) utilizan el cepillo dental tres veces al día. No así con la seda dental cuya frecuencia es mucho menor, encontrándose que hay un porcentaje importante que no la usa ( $n=37 ; 36,6 \%)$.

Se definieron once (11) variables relacionadas con la adherencia (tabla 4); por la revisión de literatura se encontró que varios estudios se refieren al cumplimiento de las citas asignadas durante el tratamiento como una de las variables más determinantes de dicho fenómeno o condición. En el análisis univariado, el 86,1% ( $n=87$ ) contestó seguir las recomendaciones adicionales de cuidado bucal dadas por el especialista, pero solo el 57,4\% (58) las recuerdan.

Se indagó por la asistencia a las citas en el día indicado; un alto porcentaje (89,1%; n=90) dijo que asistía a ellas según orientación del clínico. Los pacientes contestaron negativamente (92,1\%) cuando se les preguntó si al encontrar mejoría dejaban de asistir a la consulta, lo que indica un alto cumplimiento al encuentro con el ortodoncista.

Tabla 4. Variables que miden adherencia

\begin{tabular}{|c|c|c|c|}
\hline Variables & & $\mathbf{n}$ & Frec. relativa (\%) \\
\hline \multirow{3}{*}{ Recomendaciones adicionales de cuidado bucal } & Sí & 87 & 86,1 \\
\hline & No & 13 & 12,9 \\
\hline & SD & 1 & 1 \\
\hline \multirow{2}{*}{ Recuerda dichas indicaciones } & Sí & 58 & 57,4 \\
\hline & No & 43 & 42,6 \\
\hline \multirow{2}{*}{ Asistencia a citas día indicado } & Sí & 90 & 89,1 \\
\hline & No & 11 & 10,9 \\
\hline \multirow{2}{*}{ Inasistencia si se considera bien } & Sí & 8 & 7,9 \\
\hline & No & 93 & 92,1 \\
\hline
\end{tabular}




\begin{tabular}{lccc}
\hline \multicolumn{1}{c}{ Variables } & & $\mathbf{n}$ & Frec. relativa (\%) \\
\hline \multirow{2}{*}{ Consulta inmediata pérdida aditamentos } & Si & 46 & 45,5 \\
\cline { 2 - 4 } & No & 55 & 54,5 \\
\hline Olvida aditamentos por viaje o fuera de casa & Sí & 27 & \\
\cline { 2 - 4 } & No & 41 & 46,7 \\
\cline { 2 - 4 } & $\begin{array}{c}\text { No } \\
\text { aplica* }\end{array}$ & 33 & 32,7 \\
\hline
\end{tabular}

\begin{tabular}{lccc}
\hline \multirow{2}{*}{ Consulta oportuna por molestias } & Si & 65 & 64,4 \\
\cline { 2 - 4 } & No & 36 & 35,6 \\
\hline
\end{tabular}

\begin{tabular}{lccc}
\hline \multirow{2}{*}{ Dificultades con el tratamiento } & Si & 22 & 21,8 \\
\cline { 2 - 4 } & No & 79 & 78,2 \\
\hline
\end{tabular}

Recuerda fechas próxima consulta

\begin{tabular}{lll} 
Si & 86 & 85,1 \\
\hline No & 15 & 14,9 \\
\hline
\end{tabular}

* No se asignaron aditamentos extra

Fuente: Elaboración propia

Se preguntó a los entrevistados por el tipo de hábitos que tenían; el 64,3 \% ( $n=65)$ contestaron no tener ningún hábito. Quienes respondieron afirmativamente (35,7\%; $\mathrm{n}=45$ ) señalaron morder lapiceros o lápices, empuje lingual y apretar los dientes.

Cuando se indagó si habían recibido instrucciones sobre la manera de conservar el tratamiento, respondieron afirmativamente el 86,1 \% ( $n=87)$ de los encuestados; quiere decir que un alto porcentaje tuvo instrucciones sobre cuidado bucal. Aunque en menor porcentaje respondieron negativamente se puede considerar que todavía hay dificultades en la comunicación especialista-paciente, lo cual tiene necesariamente implicaciones en la adherencia.

El 57,4\% ( $n=58)$ de las personas recuerdan las instrucciones dadas por el clínico y las que más recuerdan son los cepillos especiales, el uso del kit de ortodoncia, asistir a las citas programadas, usar la cera sobre los brackets y las medidas de higiene bucal, principalmente. En menor porcentaje (43\%; $n=44)$ no recuerdan las instrucciones, dato que llama la atención porque la razón calculada indica que un poco más de 1 persona las recuerda por 1 que no las recuerda (1,3:1). Este hallazgo es importante a la hora de diseñar estrategias educativas que permitan mejorar la adherencia a los tratamientos. 


\section{Análisis bivariado}

En este estudio con este tamaño de muestra (que no es despreciable), no se encontró asociación estadísticamente significativa $(p>0,05)$ entre las variables relacionadas con la adherencia con respecto a las variables sociodemográficas como sexo $\left(\right.$ Chi $^{2}$ de Pearson $\left.=2,74 ; d f=2 ; p=.254\right)$, procedencia $\left(C^{2} i^{2}\right.$ de Pearson 12,$4 ; d f=12$; $p=.412$ ), tipo de aparatología (Chi ${ }^{2}$ de Pearson=6,61; $\left.d f=6 ; p=.358\right)$ y estrato $\left(\right.$ Chi $^{2}$ de Pearson=1,48; $d f=2 ; p=.477$ ). Se realizó prueba ANOVA para comparar la edad de los encuestados respecto a su nivel de adherencia y tampoco se encontraron diferencias significativas $(f=, 711 ; p=.494)$.

\section{Discusión}

En este trabajo se hace especial hincapie̋ en la necesidad de distinguir el término "adherencia" del término "cumplimiento". Este último concepto ha sido cuestionado por cuanto se considera que el paciente tiene un rol pasivo en su tratamiento, limitándose a cumplir lo prescrito por el clínico. La adherencia en cambio resalta tanto la participación activa del paciente como la responsabilidad del clínico para crear un clima de diálogo que facilite la toma de decisiones compartidas (32).

La literatura describe que la relación entre el paciente y el proveedor de servicios de salud (ya sea médico, enfermera u otro profesional de la salud) debe ser una sociedad que se base en las habilidades de cada uno, para lograr un entendimiento que posibilite ganancias de ambas partes; para el profesional porque un paciente reticente al tratamiento deteriora la relación; para el paciente, porque puede no alcanzar los resultados esperados (33).

Un determinante importante para la adherencia es la calidad de la relación clínico-paciente; Sabaté (2003) (34) expresa que relaciones de tratamiento eficaces se caracterizan por un ambiente en el que se exploran los medios terapéuticos, se negocia el régimen, se discute la adherencia y se planea el seguimiento. Por tanto, si hay poca comunicación entre las partes y el paciente no asume la importancia de su tratamiento, puede decirse que alrededor del 70 \% no es adherente, lo que indica la necesidad de mejorar la educación de los profesionales y la participación de los pacientes.

El incumplimiento terapéutico constituye un importante problema asistencial que puede afectar la salud de los pacientes, los resultados finales, y es una de las posibles causas del fracaso de los tratamientos (31). Además podría dar una falsa impresión al médico (u otro proveedor de atención de salud) acerca de la eficacia del régimen instaurado o prescrito (35). 
La OMS enumera algunos riesgos que enfrentan quienes no se adhieren a los tratamientos y son muy ilustrativos sobre las consecuencias; esto en el caso de adherencia a medicamentos, principalmente en enfermedades crónicas como la diabetes, la hipertensión arterial y el cáncer (9). En el caso de los tratamientos ortodóncicos podría asimilarse que la no adherencia retrasa el tratamiento o conduce al abandono con consecuencias negativas para el paciente.

Experiencias como los tratamientos antihipertensivos $(5,21,22)$ y antituberculosos (36) indican pobres resultados en la farmacoterapia por los tiempos de consumo de los medicamentos, las reacciones adversas, la tolerancia del paciente y la comprensión de su proceso mórbido, a la eficacia de la medicación, a los factores económicos, entre otros.

Es importante recordar una de las clasificaciones de la no adherencia: "no adherencia intencionada" y "no adherencia no intencionada" (la intención del paciente no se cumple por ciertas dificultades, como los olvidos (37). En este estudio se encontró que el $85 \%$ de los encuestados tenían presente su próxima cita y por el contrario el $15 \%$ no se acordaba de ésta.

En general, se estima que un $40 \%$ de los pacientes no cumple con las recomendaciones terapéuticas. En el caso de enfermedades agudas, la tasa de incumplimiento llega aproximadamente al $20 \%$, mientras que en las enfermedades crónicas alcanza el $45 \%$. Cuando los regímenes terapéuticos consisten en cambios de hábitos o estilos de vida, el incumplimiento es más elevado (27). En la presente investigación más de la mitad de los entrevistados recuerda las instrucciones dadas por el clínico; sin embargo, un porcentaje no despreciable no sigue estas recomendaciones.

La adherencia al tratamiento se estima generalmente como una variable binaria cumplidor/no cumplidor, con una amplia variación en los criterios utilizados. Cuando el método utilizado para estudiarlo permite su valoración cuantitativa, se considera cumplidor si se toma el $100 \%$ de la dosis recomendada, aunque se admiten variaciones, como observar el tratamiento por encima del 75-80 \%, o entre el 80 y el $110 \%$ de la dosis prescrita. Se considera entonces incumplidores a los que toman dosis menores al valor inferior e hipercumplidores a quienes lo hacen por encima del valor superior (28).

Cuando se ha estudiado el periodo de seguimiento, se puede distinguir entre incumplimiento parcial (adherencia en algunos momentos), esporádico (cuando se incumple de forma ocasional), secuencial (cuando el paciente deja el tratamiento durante periodos en que se encuentra bien y lo restaura cuando aparecen síntomas), cumplimiento de bata blanca (si sólo se adhiere cuando está cercana una visita médica) y, finalmente, incumplimiento completo (cuando el abandono del tratamiento 
es de forma indefinida) (27). En este estudio más de un tercio de la población puede considerarse como adherente, por las respuestas a las variables que se asociaron con alta adherencia, no obstante, una proporción importante de ellos tuvieron adherencia media y muy pocos ninguna adherencia.

\section{Conclusiones}

El concepto de adherencia valora de manera integral el comportamiento frente a un tratamiento o recomendaciones necesarias para el éxito del mismo, tanto de los pacientes como del clínico, mientras que la cooperación tiene en cuenta variables relacionadas sólo con el seguimiento del paciente a las recomendaciones del prescriptor mostrando un papel pasivo durante el tratamiento.

En este estudio los factores sociodemográficos como edad, sexo, procedencia y persona responsable del pago del tratamiento, no tuvieron una relación estadísticamente significativa con la adherencia al tratamiento de ortodoncia $y$, aunque se considera que el tamaño de muestra es solvente, se recomienda realizar estudios con una muestra más significativa para corroborar lo anterior.

Puede decirse que el nivel de adherencia de los pacientes al tratamiento de ortodoncia no fue satisfactorio, por tanto, es necesario redoblar esfuerzos para mejorar un indicador tan importante para el bien de los pacientes y la imagen institucional.

\section{Limitaciones}

Entendiendo la adherencia como un fenómeno complejo y multicausal que no depende solamente del paciente, es importante profundizar en aspectos referentes al servicio, especialmente la comunicación paciente-clínico porque de ello depende el feliz término de un tratamiento que es largo, incómodo y costoso.

\section{Referencias}

1. Aberastury A, Knobel M. La adolescencia normal, un enfoque psicoanalítico. México: Paidós Educador; 2004. 79 p.

2. Bergkulla N, Hänninen H, Alanko O, Tuomisto M, Kurimo J, Miettinen A, et al. Introduction and assessment of orthognathic information clinic. Eur J Orthod. [Internet]. 2017; [citado 23 de mayo de 2017] 39(6): 660.64. doi: https://doi.org/10.1093/ejo/cjx025 
Adherencia al tratamiento ortodóncico de pacientes adolescentes atendidos en una institución docente asistencial de Medellín. Estudio transversal

3. Abreu LG, Melgaço CA, Abreu MHNG, Lages EMB, Paiva SM. Agreement between adolescents and parents or caregivers in rating adolescents' quality of life during orthodontic treatment. Am J Orthod Dentofacial Orthop. [Internet]. 2015;148(6):1036-1042. doi: https://doi.org/ 10.2319/092214-681.1

4. Abreu LG, Melgaço CA, Abreu MHNG, Lages EMB, Paiva SM. Parent-assessed quality of life among adolescents undergoing orthodontic treatment: a 12-month follow-up. Dent Press J Orthod. [Internet]. 2015;20(5):94-100. doi: http://dx.doi.org/10.1590/2177-6709.20.5.094-100.oar.

5. Tapia JA. Adhesion al tratamiento farmacologico antihipertensivo en el hospital de Chaiten 2005. [Tesis de grado]. [Chile]: Escuela de Enfermería Austral De Chile; 2006. Recuperado en: http://cybertesis.uach.cl/tesis/uach/2006/fmt172a/doc/fmt172a.pdf

6. Cabrera MA, Gómez Gonzales KA. Agencia de autocuidado y su relación con la adherencia al tratamiento en funcionarios universitarios con factores de riesgo cardiovascular modificable. Manizales, Colombia: Universidad Católica de Manizales; 2014

7. Badri P, Saltaji H, Flores-Mir C, Amin M. Factors affecting children's adherence to regular dental attendance. J Am Dent Assoc. [Internet]. 2014;145(8):817-28. doi: 10.14219/jada.2014.49

8. Kontz M. A proposed model for assessing compliance within the unitary man/human framework based on an analy- sis of the concept of compliance. Philadelphia; 1991. 10 p.

9. Rodríguez AL, Gómez AM. Influential factors in adherence to treatment in patients with cardiovascular risk. Av. enferm. 2010; XXVIII(1): 63-71.

10. Bukhari OM, Sohrabi K, Tavares M. Factors affecting patients' adherence to orthodontic appointments. Am J Orthod Dentofac Orthop Off Publ Am Assoc Orthod Its Const Soc Am Board Orthod. 2016 Mar; 149(3): 319-24. doi: 10.1016/j.ajodo.2015.07.040.

11. Aljabaa A, McDonald F, Newton JT. A systematic review of randomized controlled trials of interventions to improve adherence among orthodontic patients aged 12 to 18. Angle Orthod. [Internet]. 2015; 85(2): 305-3013. doi: 10.2319/031214-184.1

12. Osterberg L, Blaschke T. Adherence to medication. N Engl J Med. [Internet]. 2005; 353(5): 487-97. doi: 10.1056/NEJMra050100

13. Kgatlwane J, Ogenyi R, Ekezie C. Factors that facilitate or constrain adherence to antiretroviral therapy among adults at four public health facilities in Botswana: a pre-intervention study. World Health Organization; 2005. 
14. Burudpakdee C, Khan ZM, Gala S, Nanavaty M, Kaura S. Impact of patient programs on adherence and persistence in inflammatory and immunologic diseases: a meta-analysis. Patient Prefer Adherence. [Internet]. 2015; 9: 435-448. doi: 10.2147/PPA.S77053

15. Conn VS, Ruppar TM, Enriquez M, Cooper PS, Chan KC. Healthcare provider targeted interventions to improve medication adherence: systematic review and meta-analysis. Int J Clin Pract. [Internet]. 2015; 69(8): 889-899. doi: 10.1111/ijcp .12632

16. Carvajal C, Sierra A, Cárdenas J. Evaluación de los cambios en la cooperación de pacientes tratados en 2 fases de Tratamiento Ortodóntico (Ortopedia-Ortodoncia). Medellín, Colombia: CES; 2013.

17. Buis L, Hirzel L, Dawood RM, Dawood KL, Nichols LP, Artinian NT, et al. Text Messaging to Improve Hypertension Medication Adherence in African Americans From Primary Care and Emergency Department Settings: Results From Two Randomized Feasibility Studies. JMIR MHealth UHealth. [Internet]. 2017; 5(2):e9. doi: 10.2196/mhealth.6630.

18. Ribeiro MN, Pimentel MIF, Schubach A de O, Oliveira R de VC de, Teixeira JL, Leite MP da $S$, et al. Factors associated to adherence to different treatment schemes with meglumine antimoniate in a clinical trial for cutaneous leishmaniasis. Rev Inst Med Trop São Paulo. [Internet]. 2014; 56(4): 291-296. doi: 10.1590/S0036-46652014000400004

19. Joury E, Marcenes W, Johal A. The role of psychosocial factors in predicting orthodontic treatment outcome at the end of 1 year of active treatment. Eur J Orthod. [Internet]. 2013; 35(2): 205-215. doi: 10.1093/ejo/cjr111

20. Artacho R, Prados J, Barnestein P, Aguiar C, García A, Leiva F. Cumplimiento terapéutico en pacientes con Hipertensión Arterial. ¿Ha cambiado con el paso del tiempo? Med fam Andal. 2012; 13(2): 95-106.

21. 21. Perseguer-Torregrosa Z, Orozco-Beltrán D, Gil-Guillen VF, Pita-Fernandez S, CarrataláMunuera C, Pallares-Carratalá V, et al. Magnitude of pharmacological nonadherence in hypertensive patients taking antihypertensive medication from a community pharmacy in Spain. J Manag Care Spec Pharm. [Internet]. 2014; 20(12): 1217-25. doi: 10.18553/jmcp. 2014.20.12.1217

22. Demoner MS, de Paula Ramos ER, Ramos Pereira E. Factors associated with adherence to antihypertensive treatment in a primary care unit. Acta Paul Enferm. [Internet]. 2012; 25(1): 27-34. doi: http://dx.doi.org/10.1590/S0103-21002012000800005 
Adherencia al tratamiento ortodóncico de pacientes adolescentes atendidos en una institución docente asistencial de Medellín. Estudio transversal

23. Ben AJ, Neumann CR, Mengue SS. The Brief Medication Questionnaire and Morisky-Green test to evaluate medication adherence. Rev Saúde Pública. [Internet]. 2012;46(2):279-89. doi: http://dx.doi.org/10.1590/S0034-89102012005000013

24. Haynes RB, Taylor DW, Sackett DL, Gibson ES, Bernholz CD, Mukherjee J. Can simple clinical measurements detect patient noncompliance? Hypertension. 1980; 2(6): 757-764.

25. Morisky DE, Green LW, Levine DM. Concurrent and predictive validity of a self-reported measure of medication adherence. Med Care. 1986 Jan; 24(1): 67-74.

26. Martínez-Pabón MC, Duque-Agudelo L, Díaz-Gil JD, Isaza-Guzmán DM, Tobón-Arroyave SI. Comparison of the effect of two sugar-substituted chewing gums on different caries- and gingivitis-related variables: a double-blind, randomized, controlled clinical trial. Clin Oral Investig. [Internet]. 2014; 18(2): 589-98. doi: 10.1007/s00784-013-0989-0

27. Vilana Pérez C, González Javier F, Ordoñana Martín J. Adherencia al tratamiento. Una revisión desde la perspectiva farmacéutica. Pharm Care Esp. 2012; 14(6): 249-255.

28. Palop V, Martínez F. Adherencia al tratamiento en el paciente anciano. Inf Ter Sist Nac Salud. 2004; 28(5): 113-120.

29. García-Pérez LE, Álvarez M, Dilla T, Gil-Guillén V, Orozco-Beltrán D. Adherence to Therapies in Patients with Type 2 Diabetes. Diabetes Ther. [Internet]. 2013; 4(2): 175-94. doi: 10.1007/ s13300-013-0034

30. Pedersini R, Isherwood G, Vietri J. Harmonizing measurement of adherence across the 4-item and 8-item morisky medication adherence scale using cross-sectional data from patients treated for irritable bowel syndrome (ibs). In Dublin, Ireland. [Internet]. 2013. doi: https://doi.org/10.1016/j.jval.2013.08.1719

31. Rodriguez Chamorro MA, García E, Amariles P, Rodriguez A, Faus MJ. Revisión de tests de medición del cumplimiento terapéutico utilizados en la práctica clínica. Aten Primaria. 2008; 40(8): 413-417.

32. Pérez-García R, Rodríguez-Benítez P. Adherencia al tratamiento con carbonato de lantano. Nefrologia Sup Ext. [Internet]. 2013; 4(2): 35-43. doi: 10.3265 /NefrologiaSuplementoExtraor dinario.pre2013.Mar.11989 
33. World Health Organization, E-libro C. Adherencia a los tratamientos a largo plazo pruebas para la acción. [Internet]. Ginebra: Organización Mundial de la Salud; 2004 [citado 21 de marzo de 2015]. Recuperado en: http://site.ebrary.com/id/10268791

34. Culig J, Leppée M. From Morisky to Hill-bone; self-reports scales for measuring adherence to medication. Coll Antropol. 2014; 38(1): 55-62.

35. Alkatheri AM, Alyousif SM, Alshabanah N, Albekairy AM, Alharbi S, Alhejaili FF, et al. Medication adherence among adult patients on hemodialysis. Saudi J Kidney Dis Transplant Off Publ Saudi Cent Organ Transplant Saudi Arab. [Internet]. 2014;25(4): 762-8. doi: 10.4103/1319-2442.134990

36. Morisky DE, Malotte CK, Choi P, Davidson P, Rigler S, Sugland B, et al. A patient education program to improve adherence rates with antituberculosis drug regimens. Health Educ Q. 1990; 17(3): 253-267.

37. Miguel JAM, Sales HX, Quintão CC, Oliveira BH, Feu D. Factors associated with orthodontic treatment seeking by 12-15-year-old children at a state university-funded clinic. J Orthod. [Internet]. 2010; 37(2): 100-106. doi: 10.1179/14653121042957 